\title{
Virtual Guide for Tourist
}

\author{
Aniket P. Chaudhari ${ }^{1}$, Akshay S. Bhargude ${ }^{2}$, Ameya J. Joshi ${ }^{3}$, Tushar D. Anekar ${ }^{4}$ \\ Student, Department of Computer Engineering, NBN Sinhgad School of Engineering, Pune, India ${ }^{1,2,3,4}$
}

\begin{abstract}
An important aspect in the tourism is to provide relevant and appropriate information about the places and the locations. Tourists may not read all information if large amount of information is provided. Hence, there is a waste of time in finding appropriate information about tourist places. Hence, it is important to provide meaningful, appropriate information. This paper describes the development of an application for smart phones, so that the tourists can carry and use application wherever they travel. In this paper, we are discussing implementation of a tourist application which will provide accurate, relevant information with appropriate recommendations to the user. A complete system will be implemented in giving precise information to the users, in a language which is universally accepted. The main focus will be on giving recommendations to the user based on tourist's behaviour. Also information about tourist places will be provided in text as well as speech form. This described here will use a wireless technology, classification technique, TTS (Text-To-Speech) to give relevant data so that user will be able to use it in many locations to give directions and information.
\end{abstract}

Keywords: Context Aware System, GPS, User Profiling, TTS.

\section{INTRODUCTION}

Tourism is well-known and one of the important roots of revenue all over the world. Tourism not only provides revenue to the country but also provides employment for many of the needy. Traditional tourism information systems which dependent on the PC and other terminal equipment, can only meet the general inquiry of tourism attraction, such as the introduction and tours, rather than the real-time weather information etc. However, increasingly improvements are being made to existing technologies. There are many systems which can provide geographical data but primarily GIS (Geographic Information Systems) is one of the best. Mobile tour guide systems has been improved due to GIS. The increase in the number of smart phones has enabled sensors to capture the user's information. Availability of precise information about the place and the location must be considered as key factor to make tourist's trip satisfactory.

Tourism is an industry which is present with abundant amount of data therefore there is heavy dose of data on tourists which can sometimes result in information overload. A major problem caused as a result of this is that both of these categories (relevant information and irrelevant information) of tourists are almost certainly not getting an experience to match their particular interests. Many a times a huge group of people are guided by a single guide who also narrates irrelevant information to the tourists. Now a day in the field of context-aware mobile applications a user can query what attractions are 'nearby' using the phone's current location [2]. However, outcomes of this query are returned with ascending order of distance. Either little or no processing takes place to personalize the results to the user's choices. Tourists are of different backgrounds and varied age differences, motivation, level of affluence and activities. The virtual guide deals with the development of a flexible system for use in museums, forts, monuments and hill stations. The typical virtual guide user will help him to access data appropriate to the tourist's places.
The idea of context has a centre role in virtual guide. This paper discusses to provide appropriate facilities to the user for tourism. The provision of context aware services is based on visitor location awareness which results into provision of context aware services. Based on this knowledge, the system will be able to adjust the context content and services (location-based services). Particularly, based on the user profile, the content is adjusted. Bayesian network will help in such processes. Based on trained Bayesian network, the application will then return points of significant interest to the tourist based on their current personalization settings.

\section{KEY FACTORS}

\section{A. Context Awareness}

Information which characterizes the situation of an entity is called as context. An entity can be an object, person, or place which is distinct and hence describes pertinent interaction between a user and an application. Contextawareness refers to a system that utilizes context to provide suitable information, facilities to the user. While designing perceptive applications it is necessary to take attainable context into account for smooth and continuous interaction between user and the surroundings.

Context is provided by utilizing various sensors such as GPS receiver, Accelerator and Compass [2]. Nevertheless, these raw sensor readings are not able to deliver significant context data alone. For this purpose, they require access to web service APIs which can read raw sensor data passed from the device and return meaningful information [2]. Reverse geocoding, a process, is an example of web services which uses GPS co-ordinates to detect rough address. This type of information can be used in the personalization process to provide more relevant information to the user.

This also refers to eliminating unnecessary and lowpriority information before showing it on the screen. 
Location will ultimately be important to the user as they are keen to know various tourist attractions that are near their current location [2]. The application will only be able to suggest those attractions which are currently open if it is combined with time. This provides another level of intelligence to the application from the user's point of view. Time span can be used to calculate the amount of time that a user stays at each attraction [2]. This information could show interests of tourists in specific attraction. APIs are capable in providing up to the minute weather conditions for the user's location. Appropriate suggestions will be given if these weather conditions are not fair for visiting outdoor attractions. For the current context, tourist themselves will be one of the main contributors [2]. Personalization would help to ensure that the given suggestions are more appropriate to the current user. However, it is also important when generating personalization data implicitly that the user remains in control. This can be facilitated by allowing the user to explicitly change any incorrect assumptions made for personalization purposes [4].

\section{B. User Profiling}

The recommendations given by context-aware mobile applications are better and appropriate. Once an application has been used for some time, it is very much possible to absolutely learn the user's behaviour. Bayesian techniques helps in learning user's behaviour. Nevertheless, the initial loading of an application shows that there will be no usage history to decide the context of the user [1]. In such case, initial assumptions are made. This process is known as user profiling. User profiling can be achieved using social media. Social media helps in deciding user's 'Family Cycle'. An efficient way of determining the user's 'Family Cycle' stage is using social network information to make an informed decision [5], as shown in Table 1.

\begin{tabular}{|l|l|}
\hline Family Cycle Status & Measurement \\
\hline Married no children & Age $<55$, married and no children \\
\hline Full nest I & $\begin{array}{l}\text { Age }<40, \text { married and children } \\
\text { present }\end{array}$ \\
\hline Full nest II & $\begin{array}{l}\text { Age }>40, \text { married and children } \\
\text { present }\end{array}$ \\
\hline Empty nest & Age $>55$, married and no children \\
\hline Single parents & $\begin{array}{l}\text { All ages, unmarried and children } \\
\text { present }\end{array}$ \\
\hline Single & $\begin{array}{l}\text { Age }<55, \text { unmarried and no } \\
\text { children }\end{array}$ \\
\hline Solitary & $\begin{array}{l}\text { Age }>55, \text { unmarried and children } \\
\text { absent }\end{array}$ \\
\hline Others & All others \\
\hline
\end{tabular}

Table 1: Family Life Cycle Stages \& Measurement [6]

Purpose for the travel is also another parameter that influences travel behaviour of the user. Hence, 'Family Cycle' alone is not sufficient for determining travel behaviour of the user. Travel behaviour can be divided into categories such as business, educational, holiday. This would not be something that could be inferred automatically with any real accuracy [6]. There may be the case that the application asks the user to select purpose of travel from a list when first launching the application in a new destination [2]. It would be crucial aspect that the user has the option to change this purpose as they may stay in a destination after a business trip and take a follow on vacation [2].

\section{Bayesian Decision Making}

A Bayesian network is a probabilistic acyclic graphical model. It depicts a set of random variables and their conditional dependencies using directed acyclic graph. The Bayesian network consists of 'Nodes' which constitute probability variables. 'Directed Arcs' are used to represent influence between each linked nodes. Bayesian networks have widely been used in language processing, intelligent system. Bayesian networks is used to use evidence node in predicting whether the probability values of related nodes are greater than a predetermined threshold [2]. For recommender system which takes user context and pattern history into account, conditional probabilities must be updated dynamically. A Gradient approach can help in Bayesian networks for such updating operation.

Bayesian network decides probability of visiting of user to each type of destination. At start, there will not be any pattern history. The purpose of using a Bayesian network is to determine (based on the various types of context) how likely it is that the user will visit each type of tourist attraction [2]. At this time, Bayesian network will use 'group probability' to give the recommendations. However, with the use of application through time Bayesian network will be created for an individual and this will be used for giving recommendations. Recently visited and the level of interest assume will be parameters based on which on which recommendations will be given.

\section{IMPORTANT TECHNIQUES}

\section{A. Android Location API}

Positioning is one of the key technologies for mobile tourism scenic spots integrated system. Positioning function can generally completed by the GPS system, however, some of the mobile terminal does not provide GPS. Our system can be compatible with various types of mobile terminals, which provides accurate positioning through GPS devices and networks. The process of positioning is shown as Fig.1. First, hardware devices are scanned to determine whether there are GPS devices or whether GPS devices are open. If the GPS devices are detected as invalid, system sets the model of Location Provider into Network Provider status, otherwise into GPS Provider and then latitude and longitude can be determined by setting the listener.

These are the different classes present under Location API package to retrieve the Location information of the user.

Location Manager- The class provides access to the location service. It also provides facility to get the best Location Provider as per the criteria.

Location Provider- It's an abstract super class for location providers. A location provider generates regular reports on the geographical location of the device. 
Location Listener- This class provides call-back methods which are called when location gets changed. The listener object has to be registered with the

Location Manager - The class provides the application to choose suitable Location Provider by providing access to set of required properties of the Location Provider. Android also provide an API to access the Google maps. So with the help of the Google maps and the location APIs, the application can show required places to the user on the map.

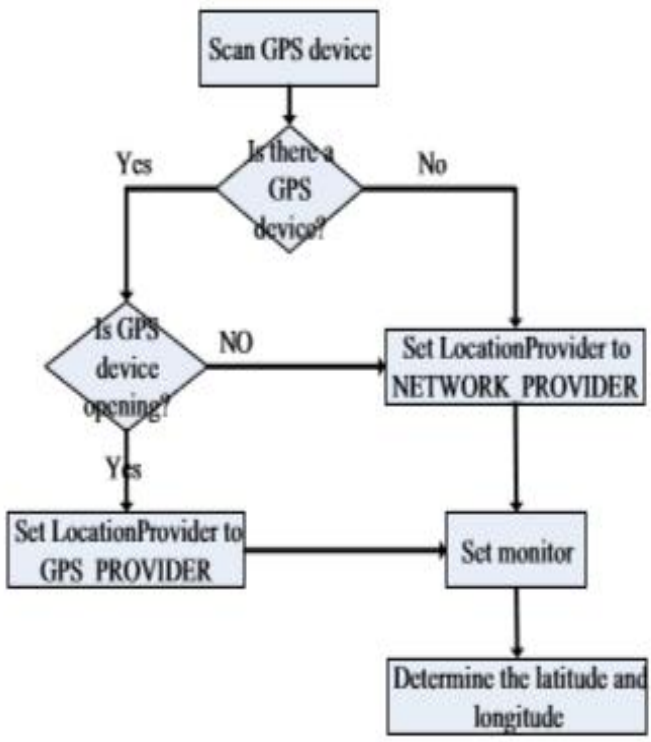

Fig 1: The process of positioning based on Android [2]

The four types of requests are available with the Google Places API there are 4 fundamental Place services available:

Place Searches - It returns an array of nearby Places based on a location defined by the user.

Place Details - It returns more specific data about a user defined Place.

Place Check-ins - It allows the request that a person has checked in to a Place.

Place Reports - It allows the users to add new locations to the Place service, and to delete Places that the application has added to the database.

\section{B. Route navigation using Android}

Real-time route navigation is one of the core functions of mobile tourism scenic spots integrated system based on Android. Attraction route navigation module searches the latitude and longitude of current location based on position location and analyses the latitude and longitude of the destination attractions through XML document. By calling Google Directions API, the route from the current position to the destination is generated, which will be saved in the temporary XML document. The system completes the path drawn on Google Map by parsing the temporary document.

The flow of attractions route navigation based on Android is shown in Fig.2.

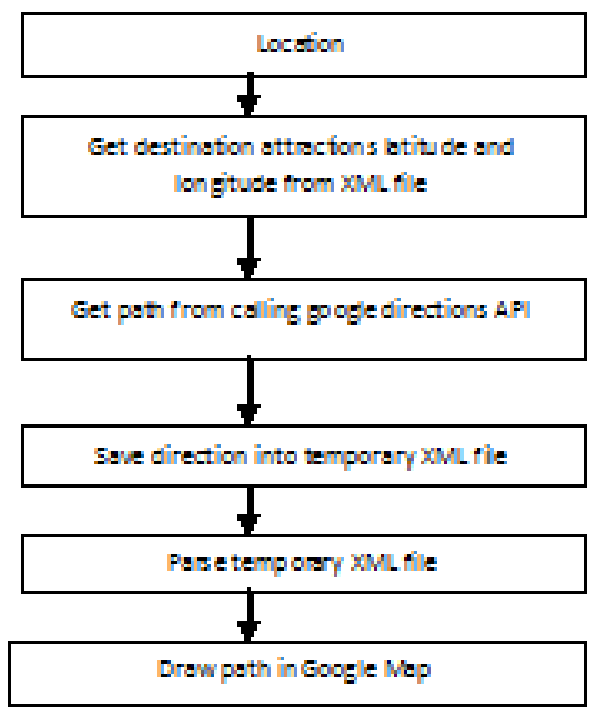

Fig2: The flow of attractions route navigation based on Android [2]

\section{TEXT TO SPEECH (TTS)}

Text-to-speech (TTS) is a speech synthesis application that is creates a spoken sound version of the text in a computer document. TTS takes text entered by user as an input, performs linguistic Formalism, operates with inference Engines and draws logical inferences and then performs digital signal processing on these inferences. General functional diagram is shown in Fig 3.

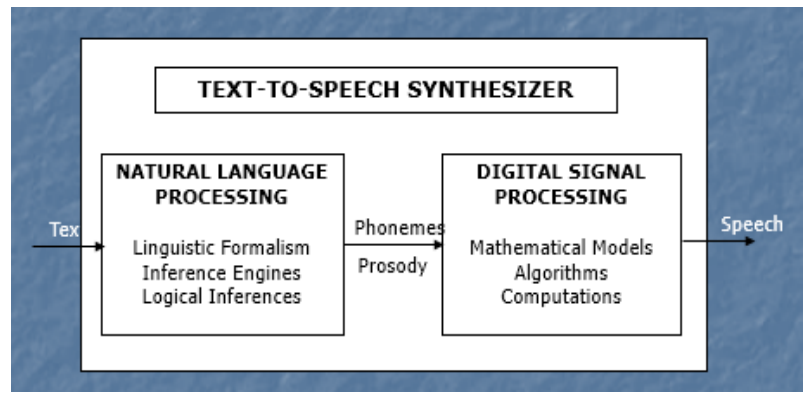

Fig 3: General functional diagram of TTS

The vice-verse of TTS is also possible.

\section{CONCLUSION}

We present our vision of flexible navigation system. We have mainly focused our discussions on providing the contextualized information to the tourists. It will help us in providing accurate information to the user. In particular, virtual guide can provide users with location-based information, which can be browsed or queried through a map. User can search the nearby attractions after he or she configures the distance between the current location and the view spots. When the user moves out of the current location, the mobile phone will automatically send its new position to the server side, and the corresponding attraction list will be received by the user.

We plan to get more real world data to get a better feel of the problem. This in a way will change the approach of tackling the problem. We intend to use different algorithms and classification techniques for it. 


\section{ACKNOWLEDGMENT}

We are really grateful to the college staff and our project guide Mrs. S. A. Chiwhane. We are also grateful to the facilities provided by our college NBN SSOE, Pune.

\section{REFERENCES}

[1] He Gang, Hu Tao. , Applied research on mobile tourism scenic spots integrated system based on Android, 2013 10th Web Information System and Application Conference., (pp. 167-169).

[2] Kevin Meehan, Tom Lunney, Kevin Curran, Aidan McCaughey. (2012), VISIT: Virtual Intelligent System for Informing Tourists.

[3] Barbara Schmidt-Belz, Nick Achim, Stefan Poslad, and Alex Zipf, "Personalized and location-based mobile tourism services," in In Proceedings of Mobile-HCI Conferected, Pisa, 2002.

[4] G Hong, J Fan, L Palmer, and V Bhargava, "Leisure Travel Expenditure Patterns by Family Life Cycle Stages," Journal of Travel \& Tourism Marketing, vol. 2, no. 18, pp. 15-30, 2005.

[5] Linas Baltrunas, Bernd Ludwig, Stefan Peer, and Francesco Ricci, "Context-Aware Places of Interest Recommendations of Mobile Users," in Human-Computer Interaction International, Heidelberg, Berlin, 2011, pp. 531-540.

[6] H Fang-Ming, L Yu-Tzeng, and H To-Kuang, "Design and implementation of an intelligent recommendation system for tourist attractions: The integration of EBM model, Bayesian Network and Google Maps," Expert Systems with Applications, vol. 39, no. 3, pp. 3257-3264, February 2012 